\section{Characteristic of Subjects Who Fail a 120-Minute Spontaneous Breathing Trial: When Minutes Are Taken Into Account}

\section{To the Editor:}

Both a failure of a spontaneous breathing trial (SBT) and extubation are markers of poor outcomes in critically ill patients. ${ }^{1,2}$ However, the determinants of risk in selected populations have not been well characterized. We read with interest the manuscript published in the Journal by Liang et $\mathrm{al},{ }^{3}$ which endeavored to characterize patients at risk of late SBT failure; however, several key considerations warrant further discussion.

First, the population in this study, as described in the first table, appears to include many subjects who could be easily weaned from the ventilator and few subjects who required long-term mechanical ventilation or were difficult to wean. Specifically, using the Weaning according to a New Definition (WIND) classification, we do not know the proportion of subjects in this study who could have been weaned on the first attempt within the first day (simple classification); within the first $2-7 \mathrm{~d}$ with 1-3 weaning attempts (difficult classification); or at least $7 \mathrm{~d}$ after the first weaning attempt or requiring $>3$ weaning attempts, or those who were never weaned (prolonged classification). ${ }^{4}$ This is likely an important consideration in liberating otherwise heterogeneous critically ill patients from invasive ventilation.

Second, the SBT technique utilized in this study (ie, pressure support with zero PEEP) is infrequently used in clinical practice, ${ }^{5}$ so these results may have limited utility to clinical practice except for selected populations (eg, patients with COPD or congestive heart failure).

Third, regarding the identified predictors of SBT failure, several factors, including the presence of chronic cardiopulmonary disease, the number of previous SBT attempts before achieving success at $30 \mathrm{~min}$, subject age, and elevated $\mathrm{P}_{\mathrm{CO}_{2}}$, were independently associated with SBT success at $30 \mathrm{~min}$ and failure at $120 \mathrm{~min}$. However, it is not clear why $\mathrm{P}_{\mathrm{aCO}}$, the rapid shallow breathing index, $\Delta \mathrm{P}_{\mathrm{aO}_{2}} / \mathrm{F}_{\mathrm{IO}_{2}}$ (T30-T0), $\Delta$ breathing frequency (T30-T0), and $\Delta \mathrm{pH}$ (T30-T0) were all independently associated with 30 min success and $120 \mathrm{~min}$ SBT failure. One is left to postulate that unmeasured variables, such as patient-related factors (eg, neuromuscular or diaphragm weakness, frailty, malnutrition, or baseline spirometry parameters) may have influenced these measures.

Fourth, with only 41 subjects who passed a 30-min SBT and failed a 120-min SBT, the data are insufficient and underpowered to generate accurate prediction scores. Questions remain regarding the optimal SBT duration and which patients may benefit from SBTs of longer duration.

As a preliminary investigation, the study by Liang et $\mathrm{al}^{3}$ highlights the need for additional studies in this area to identify the patients at risk of SBT failure, the best SBT technique to utilize in clinical practice, and the optimal SBT duration. This study could have been complemented by use of the WIND classification to characterize subjects on the basis of the number of weaning attempts and the duration of invasive ventilation. In addition, more robust baseline data and concurrent physiologic measurements may have aided in characterizing subject work of breathing and would have provided insight into reasons for SBT failure. Considering the clinical consequences associated with SBT and extubation failure and the frequency with which clinicians identify SBT candidates and conduct SBTs in clinical practice, weaning should be identified as key research priority in critical care.

Karen E A Burns Critical Care Medicine

St. Michael's Hospital and the Li Ka Shing Knowledge Institute Toronto, Ontario

Habib M R Karim

Department of Anaesthesiology and Critical Care

All India Institute of Medical Sciences Raipur, India

\section{Antonio M Esquinas \\ Intensive Care Unit \\ Hospital Morales Meseguer Murcia, Spain}

The authors have disclosed no conflicts of interest.

DOI: $10.4187 /$ respcare.06404

\section{REFERENCES}

1. Ely EW, Baker AM, Evans GW, Haponik EF. The prognostic significance of passing a daily screen of weaning parameters. Intensive Care Med 1999;25(6):581-587.

2. Esteban A, Alia I, Gordo F, Fernández R, Solsona JF, Vallverdú I, et al. Extubation outcome after spontaneous breathing trials with t-tube or pressure support ventilation. The Spanish Lung Failure Collaborative Group. Am J Respir Crit Care Med 1997; 156(2 Pt 1):459-465.

3. Liang G, Liu T, Zeng Y, Shi Y, Yang W, Yang Y, Kang Y. Characteristics of subjects who failed a 120-minute spontaneous breathing trial. Respir Care 2018;63(4): 388-394.

4. Béduneau G, Pham T, Schortgen F, Piquilloud L, Zogheib E, Jonas M, et al.; WIND Study Group and the REVA (Réseau Européen de Recherche en Ventilation Artificielle) Network: Epidemiology of weaning outcome according to a new definition: The WIND study. Am J Respir Crit Care Med 2017;195(6):772-783.

5. Burns KEA, Raptis S, Nisenbaum R, Rizvi L, Jones A, Bakshi J, et al. International practice variation in weaning critically ill adults from invasive mechanical ventilation. Ann Am Thorac Soc 2018;15(4):494502

\section{Lung Ultrasound: Just B Lines?}

\section{To the Editor:}

We enjoyed reading the interesting article by Antonio et $\mathrm{al}^{1}$ regarding the use of lung ultrasound to predict extubation in critical care subjects. Studies with neutral or negative results like this one are extremely important, in the same tenor as those with positive findings. In this era, in which pointof-care ultrasound seems to be a panacea, this article teaches us that we are treating patients, not images, and this is a concept we should always keep in mind. As a matter of fact, point-of-care ultrasound should be never used in isolation in any application related to patients in need of critical care. However, we would like to make some constructive comments about this work.

First, the assertion that lung ultrasound is not helpful prior to a spontaneous breathing trial (SBT) seems to be imprudent, primarily because this article does not study the direct effects of other alterations detected by lung ultrasound prior to SBT, such as large pleural effusions, consolidations, or diaphragmatic dysfunction, regardless of the presence or absence of B lines. ${ }^{2}$

Second, while the 4-zone lung-study protocol allows rapid scanning as noted by the authors, this is not representative of the whole picture of the lungs, espe- 